University of South Carolina

Scholar Commons

$11-2004$

\title{
Resonance-Enhanced Multiphoton Ionization for Real-Time Monitoring of Trichloroethylene formed by Degradation of Tetrachloroethylene Using Zero-Valent Zinc
}

\author{
Kui Chen \\ University of South Carolina - Columbia \\ Jack E. Pender \\ University of South Carolina - Columbia \\ John L. Ferry \\ University of South Carolina - Columbia, ferry@mailbox.sc.edu \\ S. Michael Angel \\ University of South Carolina - Columbia
}

Follow this and additional works at: https://scholarcommons.sc.edu/chem_facpub

Part of the Chemistry Commons

Publication Info

Published in Applied Optics, Volume 43, Issue 33, 2004, pages 6207-6212.

(C) Applied Optics 2004, The Optical Society.

This Article is brought to you by the Chemistry and Biochemistry, Department of at Scholar Commons. It has been accepted for inclusion in Faculty Publications by an authorized administrator of Scholar Commons. For more information, please contact digres@mailbox.sc.edu. 


\title{
Resonance-enhanced multiphoton ionization for real-time monitoring of trichloroethylene formed by degradation of tetrachloroethylene using zero-valent zinc
}

\author{
Kui Chen, Jack E. Pender, John L. Ferry, and S. Michael Angel
}

\begin{abstract}
Resonance-enhanced multiphoton ionization (REMPI) is investigated as a potential technique for realtime monitoring of selected volatile organochloride compounds (VOCs). In a proof-of-concept experiment, the progress of the reductive-degradation of tetrachloroethylene (PCE) to trichloroethylene (TCE) by zero-valent zinc was monitored by REMPI measurements performed in the headspace above the PCE solution. Two-photon resonant REMPI spectra of TCE and PCE were recorded over the wavelength range $305-320 \mathrm{~nm}$. The concentrations of PCE and TCE in the headspace were monitored by measurement of the ionization signal with 315.64- and 310.48-nm excitation for PCE and TCE, respectively. Calibration curves yielded a linear range of more than 2 orders of magnitude for both compounds. The REMPI headspace results agreed well with the solution-phase results from gas chromatography analysis, which was used for independent verification of the progress of the reaction. (c) 2004 Optical Society of America

OCIS codes: $300.6350,020.4180,260.5740,300.6360$.
\end{abstract}

\section{Introduction}

Resonance-enhanced multiphoton ionization (REMPI) is a laser ionization technique that has been used extensively as a selective ionization source for mass spectrometry. ${ }^{1-4}$ However, one can also use it directly as an analytical technique by measuring the ionization current with an electrode. In the REMPI process, a molecule is excited by a photon, or photons, whose energy is in resonance with an electronic state of the molecule. The excited molecule is then ionized by absorption of additional photons. Resonance ionization is highly efficient because of the increased lifetime of the intermediate state by as much as 6 orders of magnitude compared with that of nonresonant multiphoton ionization. ${ }^{5}$ Various excitation schemes can be used for REMPI, and these are described by the number of photons used for excitation

The authors are with the Department of Chemistry and Biochemistry, University of South Carolina, 631 Sumter Street, Columbia, South Carolina. 29208. S. M. Angel's e-mail address is angel@mail.chem.sc.edu.

Received 2 January 2004; revised manuscript received 27 July 2004; accepted 5 August 2004.

0003-6935/04/336207-06\$15.00/0

(C) 2004 Optical Society of America and ionization. For example, in the simplest resonance-ionization scheme one photon is used to excite the molecule and an additional photon to ionize it, this is the $1+1$ REMPI scheme. In higher-order excitation schemes, two or more photons are involved in the excitation and the ionization processes $(2+1$, $2+2$, etc.). For simple quantitative measurements the ionization signal can be measured directly as a voltage drop across a resistor at a single excitation wavelength. For qualitative measurements, one records a REMPI excitation spectrum by scanning the laser over a wavelength range that covers the absorption features of interest while monitoring the ionization current. The REMPI excitation spectrum provides a fingerprint of the molecule, and it offers the possibility of selective ionization against a chemically similar background. ${ }^{6}$

We are investigating REMPI for both qualitative and quantitative in situ detection of volatile organic compounds (VOCs) under ambient conditions. We are also incorporating fiber-optic strategies to deliver the excitation light to the sample area. ${ }^{7,8}$ The addition of fiber optics allows the use of REMPI for such applications as in situ monitoring of environmental pollutants and evaluation of the performance of remediation technologies such as in situ reductive degradation of $\mathrm{VOCs}$ in permeable reactive barriers. In 
previous reports REMPI was shown to be sensitive to parts-per-billion (ppb) levels of vapor-phase organic contaminants such as benzene, toluene, and xylenes. ${ }^{7,8}$ In those reports it was also shown that visible-light-transmitting optical fibers can be used to measure REMPI spectra of UV-absorbing aromatic compounds by use of a $(2+2)$ REMPI excitation scheme. ${ }^{7,8}$ In the present study we extend this research to include in situ and real-time measurements of vacuum-UV-absorbing VOCs, using a $(2+1)$ excitation scheme. We measured trichloroethylene (TCE) and tetrachloroethylene (PCE) absorption bands at 152.5-160 nm, using two-photon resonant excitation, by scanning the dye laser from 305 to 320 $\mathrm{nm}$. In this way we used REMPI to monitor, in situ, the amount of TCE in the headspace above an aqueous PCE solution to follow the degradation of PCE after addition of zero-valent zinc. This reaction was chosen because of its environmental relevance: zero-valent metals are currently employed in field studies as the active materials in permeable reactive barriers, offering a low-cost alternative to the removal of chlorinated VOCs. ${ }^{9,10}$

\section{Experiment}

\section{A. Sample Preparation}

The following chemicals were used as received without further treatment: PCE [99\%, American Chemical Society (A. C. S.) reagent, Aldrich Chemical Company], TCE (99.5\%, A. C. S. reagent, Aldrich), methanol (99.9\%, high-performance liquid chromatography grade, Fisher Scientific), Tris(hydroxymethyl)aminomethane $(99.8-100.1 \%$, A. C. S. reagent, Alfa Aesar), Zn granules (20 mesh, A. C. S. reagent, Alfa Aesar), sodium chloride (99.0\%, GR crystals, EM Science), methyl $t$-butyl ether (OmniSolv GC grade, EM Science), and 4-bromoanisole (99+\%, A. C. S. reagent, Aldrich).

We prepared PCE and TCE stock solutions (6 parts in $10^{3}$, volume to volume) by dissolving $300 \mu \mathrm{L}$ of neat PCE and TCE in methanol in a $50-\mathrm{mL}$ volumetric flask. Further dilution was made with $0.1 \mathrm{M}$ $\mathrm{NaCl}-50 \mathrm{mM}$ Tris buffer (pH 7.2) by introduction of TCE or PCE into the aqueous solution in a methanol carrier where needed. The buffer was used to maintain the $\mathrm{pH}$ of the PCE solution during the reaction with zero-valent $\mathrm{Zn}$. All calibration standards were also made with the same buffer solution. For the breakdown experiments, $40 \mu \mathrm{L}$ of $\mathrm{PCE}-\mathrm{CH}_{3} \mathrm{OH}$ stock solution was used to spike the buffer to make a 3.2 parts-in- $10^{6}$ (ppm) weight to weight PCE solution, and a load of $6 \mathrm{~g}$ of 20-mesh Zn granules was added. We prepared the calibration standards in the same way as the reaction samples by spiking $120 \mathrm{~mL}$ of 0.1 $\mathrm{M}$ NaCl-50 mM Tris buffer with aliquots of TCE or $\mathrm{PCE}-\mathrm{CH}_{3} \mathrm{OH}$ stock solution to achieve the desired concentrations.

\section{B. REMPI Experimental Setup}

A schematic diagram of the REMPI experimental setup is shown in Fig. 1. All the REMPI excitation

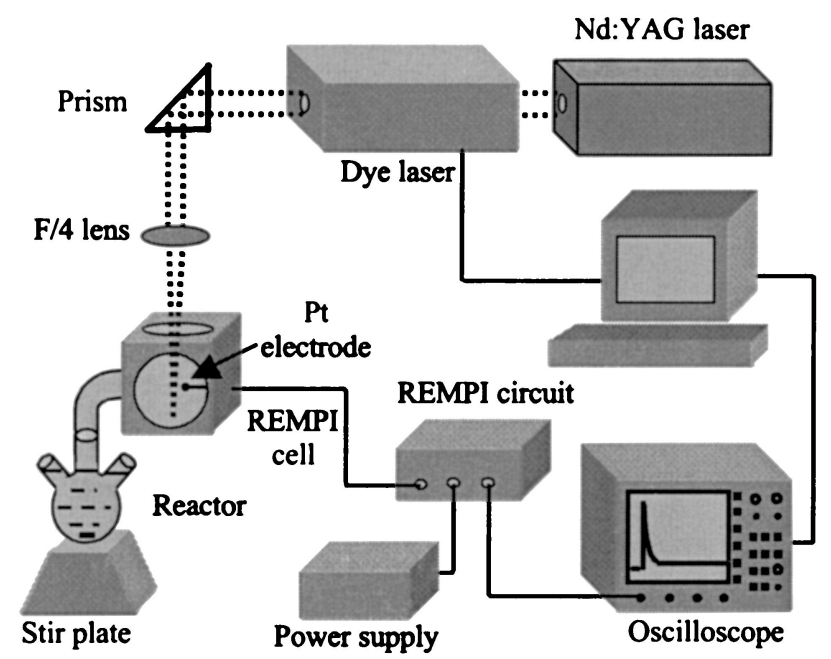

Fig. 1. Schematic diagram of the REMPI experimental setup.

spectra were measured with a Continuum (Santa Clara, California) Jaquar dye laser operated with 4-dicyanmethylene-2-methyl-6-( $p$-dimethylaminostyryl)- $4 H$-pyran (DCM) dye dissolved in ethanol. The second harmonic of a Continuum Surelite III pulsed $\mathrm{Nd}$ :YAG laser firing at a rate of $10 \mathrm{~Hz}$ was used to pump the dye laser. The output of the DCM dye was frequency doubled with an angle-tuned KDP doubling crystal, permitting spectral access to the region 305$320 \mathrm{~nm}$, which covers the two-photon absorption range of TCE and PCE. A built-in autotracker was used for automatic angle tuning of the KDP doubling crystal during the scanning of the dye laser. A typical laser pulse energy used in the experiment was $\sim 2 \mathrm{~mJ}$ at the peak of the dye curve.

The laser beam was focused directly into the REMPI cell approximately $1 \mathrm{~mm}$ away from a platinum electrode by a $10-\mathrm{cm}$ focal-length lens, and the laser power was measured continuously during a wavelength scan by a Molectron (Portland, Oregon) EPM1000 powermeter placed behind the cell. The current generated during the REMPI process was collected by the platinum electrode biased at $+500 \mathrm{~V}$, converted to into voltage by a lab-built current-tovoltage converter and a preamplifier, and measured with a LeCroy (Chestnut Ridge, New York) Model LC 534A fast digital oscilloscope. A PC running a custom Labview program was used to coordinate the operation of the dye laser, the powermeter, and the oscilloscope. Both the REMPI signal and the power of the laser beam were displayed in real time and saved to a spreadsheet file after completion of a wavelength scan.

\section{Monitoring PCE Degradation}

To demonstrate the use of REMPI to monitor the progress of a reductive degradation reaction in situ, we used the breakdown of PCE by zero-valent zinc. This reaction was chosen to simulate the VOC breakdown processes in a permeable reactive barrier with zero-valent metals as the active material. The 


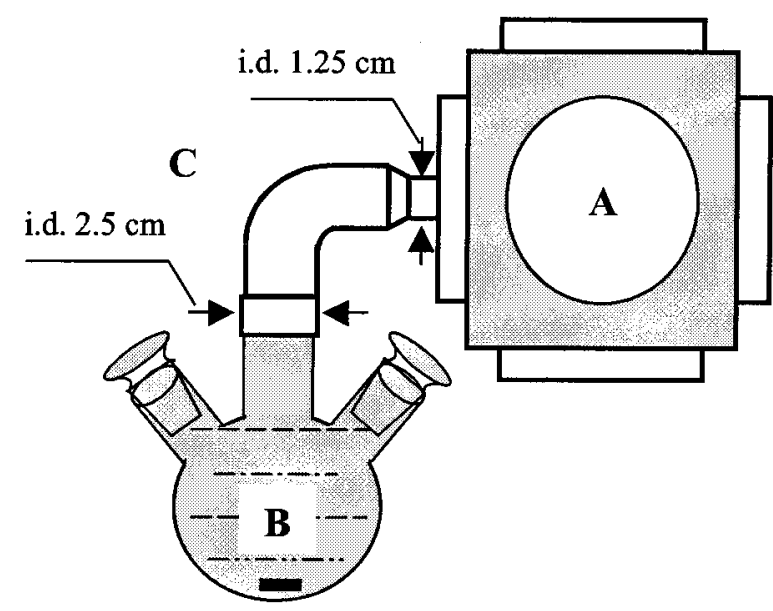

Fig. 2. Schematic diagram of the REMPI measurement system: A, detection cell; B, reactor assembly with dimensions of the REMPI cell port; $\mathrm{C}$, brass elbow connector. The total volume of the solution is $120 \mathrm{~mL}$. The headspace volume above the solution is estimated to be $250 \mathrm{~mL}$.

breakdown experiment was carried out in a threenecked flask reactor that contained $120 \mathrm{~mL}$ of the aqueous PCE solution that was attached to a sealed REMPI cell by a brass elbow connector (Fig. 2). The total headspace volume above the solution (including the volume in the REMPI cell and in the elbow connector) was estimated to be $\sim 250 \mathrm{~mL}$. In this assembly the rate of diffusion from the solution to the measurement cell was limited by a narrow point, of $1.27-\mathrm{cm}$, between the REMPI cell and the reactor. The PCE aqueous solution was allowed to equilibrate with the headspace before the $\mathrm{Zn}$ granules were added. Throughout the experiment, the suspension was agitated with a magnetic stirrer. We monitored the progress of the reaction for more than $24 \mathrm{~h}$ by recording REMPI spectra of PCE and TCE. As each REMPI measurement was made, a $250-\mathrm{uL}$ aliquot of the suspension was drawn from the flask for comparative gas chromatography (GC) analysis.

\section{Gas Chromatography Analysis}

Quantitative GC analysis was performed to confirm the REMPI results. Samples were drawn from the reactor at selected times during the breakdown experiment and extracted with methyl $t$-butyl ether with 4-bromoanisole as an internal standard. Quantitative analysis of PCE and TCE was achieved with a Hewlett-Packard (Palo Alto, California) 5890 Series II gas chromatograph equipped with a ${ }^{63} \mathrm{Ni}$ electron-capture detector. A Hewlett-Packard HP7673A autoinjector was used to deliver $1 \mu \mathrm{L}$ of samples into the injection port held at $280^{\circ} \mathrm{C}$ in split mode at a split ratio of 12:1. Deactivated glass wool was placed in the injection port liner to promote reproducible volatilization. Helium $\left(1.6 \mathrm{~mL} \mathrm{~min}{ }^{-1}\right)$ was used as the carrier gas through a DB-5MS analytical column from J\&W Scientific [length, $30 \mathrm{~m}$; inner diameter (i.d.), $0.25 \mathrm{~mm}$; deflection factor, 0.25 $\mu \mathrm{m}]$. The oven temperature was held isothermally at $60{ }^{\circ} \mathrm{C}$ for $1 \mathrm{~min}$ and then ramped at $40{ }^{\circ} \mathrm{C} / \mathrm{min}$ to $270{ }^{\circ} \mathrm{C}$ and held isothermally for $3 \mathrm{~min}$. The temperature of the electron-capture detector was maintained at $310^{\circ} \mathrm{C}$, and nitrogen was used as the make-up gas $\left(50 \mathrm{~mL} \mathrm{~min}{ }^{-1}\right)$. Automated instrument control and data acquisition were accomplished by use of HP3365 Series II Chemstation Version A.03.21 software.

\section{Results and Discussion}

\section{A. REMPI Spectra of TCE and PCE}

Figure 3 shows $(2+1)$ REMPI spectra, uncorrected for the dye gain, of vapor-phase PCE and TCE measured over a 30-ppm aqueous solution. The TCE spectrum is offset for clarity. Over this wavelength region, TCE shows bands from 307 to $313 \mathrm{~nm}$, with the most intense peak at $310.48 \mathrm{~nm}$ originating from a $\pi \rightarrow 3 d$ transition. ${ }^{11}$ The bands for PCE across the same wavelength range are much weaker and much broader, with two relatively strong peaks at 315.64 and $314.72 \mathrm{~nm}$ that correspond to $\pi \rightarrow 3 p$ transitions. ${ }^{12}$ Although PCE has much stronger REMPI bands at other wavelength regions $(343.8 \mathrm{~nm}$, for instance), ${ }^{12}$ we chose the relatively weak band at $315.64 \mathrm{~nm}$ for PCE detection in this proof-of-concept experiment. We did this because the weaker band at $315.64 \mathrm{~nm}$ had the convenience of being relatively close to the strong TCE bands, allowing the experiments to be done rapidly with a single laser dye. As can be seen from the spectra, TCE can be selectively excited at $310.48 \mathrm{~nm}$ with little interference from PCE.

\section{B. REMPI Calibration}

Calibration curves for both TCE and PCE were obtained by use of spiked aqueous standards $(0.1 \mathrm{M}$ NaCl-50 mM Tris; pH 7.2). REMPI spectra were acquired after equilibrium was reached between the solution and the headspace $(\sim 2.5 \mathrm{~h})$. For calibration, REMPI spectra were acquired in the headspace from 309.68 to $311.52 \mathrm{~nm}$ for TCE and from 315.16 to $316.8 \mathrm{~nm}$ for PCE, and the background-corrected peak intensities were plotted against the original solution-phase analyte concentrations. Calibration curves for TCE and PCE (Figs. 4 and 5, respectively) both showed linear responses of more than 2 orders of magnitude from 40 to $4000 \mathrm{ppb}$ [nanogram/gram $(\mathrm{ng} / \mathrm{g})]$. Although the precision of the measurements was not so good for PCE as for TCE, REMPI was shown to be sensitive to ppb levels of both compounds.

\section{Comparison of REMPI and Gas Chromatography Results of PCE Degradation}

Two major pathways, hydrogenolysis and reductive $\beta$-elimination of halides, ${ }^{13}$ are generally involved in the degradation of chloroethylenes by zero-valent metals. These reactions have been investigated because of their potential to reduce chloroethylenes in groundwater to the much less toxic ethylene. The reaction between zero-valent $\mathrm{Zn}$ and PCE proceeds mainly by 


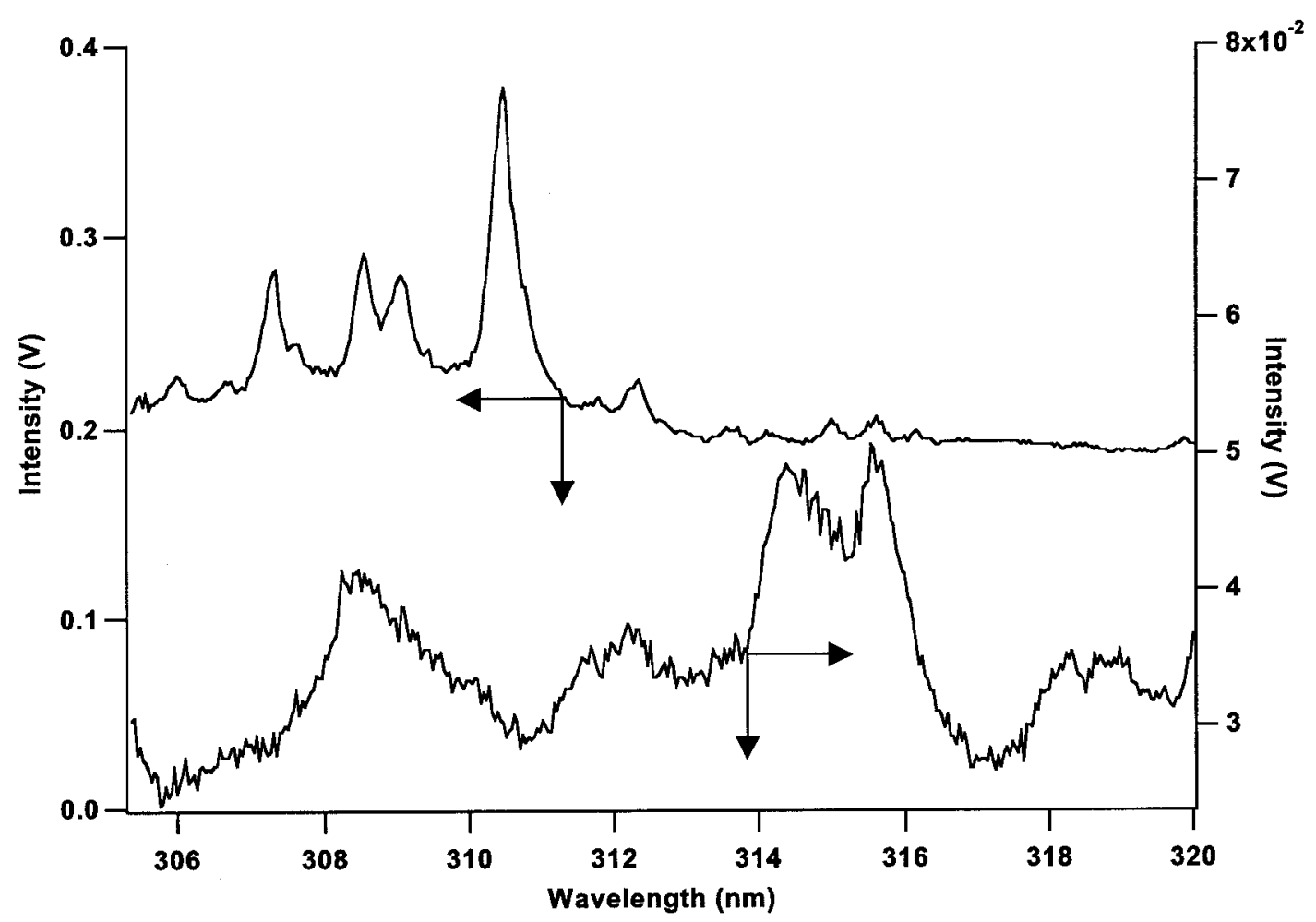

Fig. 3. $(2+1)$ REMPI spectra of top, TCE and bottom, PCE measured in the headspace above 30-ppm solutions (the TCE spectrum is offset for clarity).

hydrogenolysis and produces several intermediates en route, notably TCE $(>85 \%)$ and trans-dichloroethene (DCE) $(\sim 10 \%){ }^{11}$ As the intermediates react more slowly with zero-valent $\mathrm{Zn},{ }^{13}$ they will build up over time during PCE degradation. In our system this translates into an expected drop in PCE concentration with a concurrent increase in TCE and trans-DCE concentrations. Because no trans-DCE absorption band is available over the wavelength range studied, no effort was made to monitor trans-DCE during the reaction. REMPI spectra of TCE and PCE were taken at selected times during the reaction in the headspace

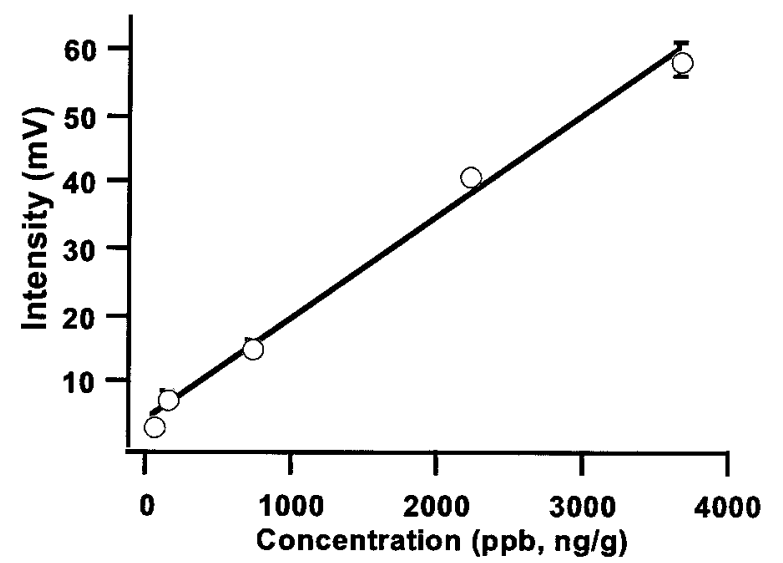

Fig. 4. Calibration of a TCE-spiked water sample. Each calibration point represents the average of three measurements at 310.48 $\mathrm{nm}$; error bars show \pm 1 standard deviation. above the reaction mixture without perturbing the matrix of the reaction. As can be seen from Fig. 6, a band corresponding to TCE (310.48 nm) appeared soon after the addition of $\mathrm{Zn}$ granules and continued to increase throughout the experiment. During the same time period we also observed a steady drop in the intensity of the PCE signal at $315.64 \mathrm{~nm}$. A control experiment with no $\mathrm{Zn}$ added showed no significant loss of PCE or production of TCE as expected under our experimental conditions.

The change in PCE and TCE concentrations during the breakdown reaction can be seen more clearly in

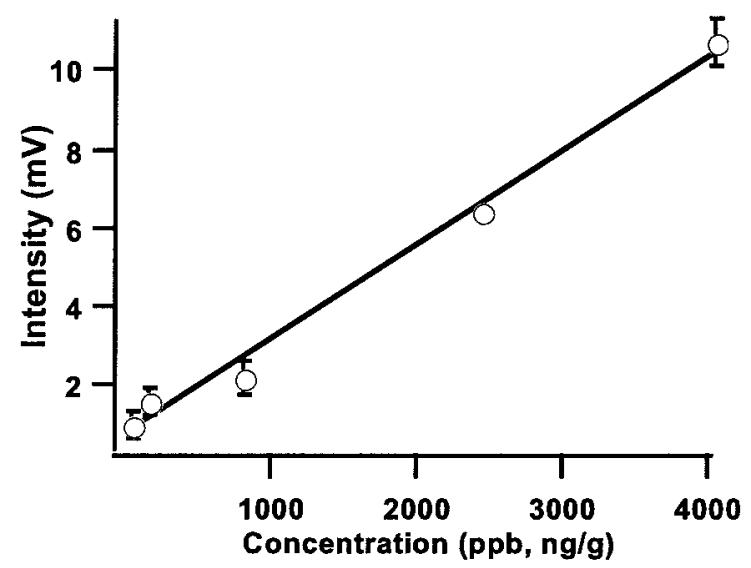

Fig. 5. Calibration of a PCE-spiked water sample. Each calibration point represents the average of three measurements at 315.64 $\mathrm{nm}$; error bars show \pm 1 standard deviation. 


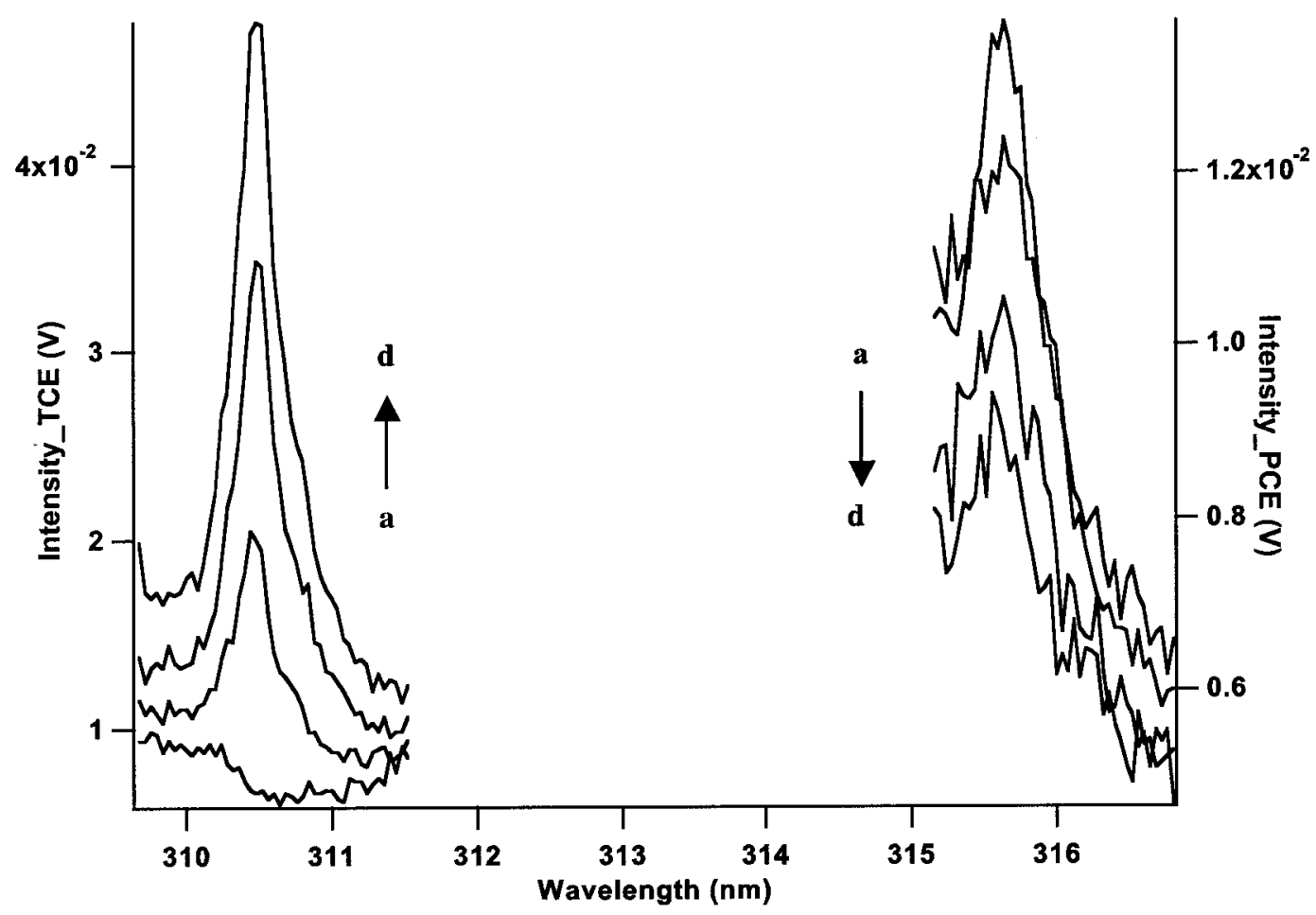

Fig. 6. REMPI spectra of left, TCE and right, PCE measured in the headspace at selected times during PCE degradation by use of zero-valent zinc: (a) before addition of $\mathrm{Zn}$, and (b) $4 \mathrm{~h}$ after, (c) $12 \mathrm{~h}$ after, and (d) $24 \mathrm{~h}$ after addition of $\mathrm{Zn}$.

Fig. 7, where the analyte concentrations measured with REMPI and GC are plotted against the reaction time. Both sets of data reveal the same general trend in TCE and PCE concentration changes. Although the results from the REMPI measurements deviate from the GC analysis in the early stage of the reaction, they tend to converge toward the end. The disparity between REMPI and GC occurs partially because the REMPI measurement is based on the vapor-phase concentration, whereas GC analysis

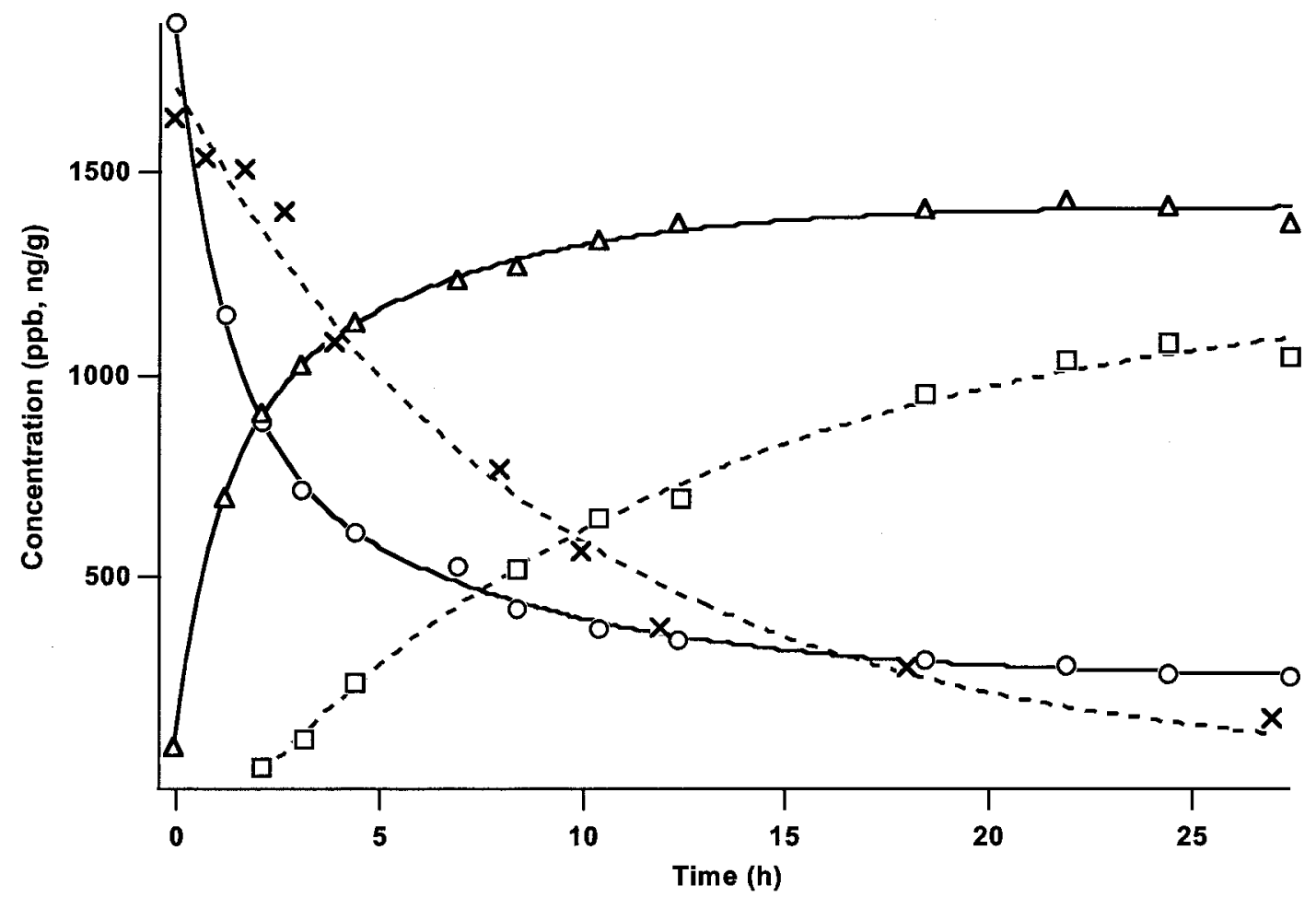

Fig. 7. PCE (open circles) and TCE (open triangles) concentrations obtained by GC (solid curves) measured with solution aliquots, and PCE (crosses) and TCE (open squares) concentrations obtained by REMPI (dashed curve) measured in the headspace above the solution. 
measures the concentration in the solution directly. The REMPI cell that we currently use was not optimized for rapid equilibration between dissolved and vapor-phase TCE and PCE. In fact, the large headspace volume and narrow constriction between the reactor and the REMPI cell made equilibration extremely slow. As a result, there is a large hysteresis in the current setup, and the change in the analyte concentration in the headspace will always lag behind the change in the solution. At the beginning of the reaction, the rate of reaction was relatively fast, as GC analysis showed a quick rise in TCE concentration and a simultaneous prompt drop in PCE concentration. As the solution-phase PCE concentration decreased, PCE concentration in the headspace dropped more slowly owing to slow diffusion back into the solution. The PCE concentration measured by REMPI was thus higher than that by GC early in the reaction when degradation proceeded at a faster pace. The concentration of TCE obtained by REMPI, however, is lower than that by GC because of the slow mass transfer from solution to the headspace. As the reaction proceeded and the PCE concentration decreased, the surface of the $\mathrm{Zn}$ granules is gradually oxidized and passivated, causing the reaction in the solution to slow down. Therefore, during the later stages when the reaction is near completion, mass transfer is better able to keep up with the concentration changes in the solution. At this point the REMPI and GC curves begin to converge.

Again, the discrepancy between REMPI and GC is probably unique to our particular cell design because of the large headspace volume and the small constriction between the flask and the REMPI cell. One could minimize this issue by optimizing the design of the REMPI cell, using a low headspace volume and maximizing the area for diffusion between the reaction vessel and the REMPI cell.

\section{Conclusions}

REMPI under ambient conditions was successfully used to monitor the degradation of PCE to TCE by zero-valent zinc. It was shown to be a promising in situ technique for VOC detection and monitoring and offers high sensitivity for quantitative measurements of $\mathrm{ppb}$ levels of chloroethylenes. With different wavelength ranges readily available by use of differ- ent dyes, selective REMPI detection of most chloroethylenes is possible with the appropriate choice of laser dye. The feasibility of using visible or near-UV photons for UV- or vacuum-UV-absorbing molecules has also been demonstrated. To explore the full potential of this technique will require further characterization of REMPI spectral features of other chlorinated VOCs, which is currently under way.

\section{References}

1. A. Clark, K. W. D. Ledingham, A. Marshall, J. Sander, and R. P. Singhal, "Attomole detection of nitroaromatic vapors using resonance-enhanced multiphoton ionization massspectrometry," Analyst 118, 601-607 (1993).

2. D. R. Nesselrodt and T. Baer, "Cyclic ketone mixture analysis using $2+1$ resonance-enhanced multiphoton ionization massspectrometry," Anal. Chem. 66, 2497-2504 (1994).

3. C. H. Sin, R. Tembreull, and D. M. Lubman, "Resonant 2-photon ionization spectroscopy in supersonic beams for discrimination of di-substituted benzenes in mass-spectrometry," Anal. Chem. 56, 2776-2781 (1984).

4. D. R. Nesselrodt, A. R. Potts, and T. Baer, "Observation of ethyl-substituted cyclohexanone and cyclopentanone rotamers using resonance-enhanced multiphoton ionization spectroscopy,” J. Phys. Chem. 99, 4458-4465 (1995).

5. M. B. Robin, "Multiphoton fragmentation and ionization," Appl. Opt. 19, 3941-3947 (1980).

6. P. M. Johnson, "Molecular multiphoton ionization spectroscopy," Acc. Chem. Res. 13, 20-26 (1980).

7. R. C. Chinni, "In-situ characterization using pulsed laser systems and hyperspectral imaging," Ph.D. dissertation (University of South Carolina, Columbia, S. C. 2002).

8. B. M. Cullum, S. K. Shealy, and S. M. Angel, "Fiber-optic resonance-enhanced multiphoton ionization probe for in situ detection of aromatic contamination," Appl. Spectrosc. 53, 1646-1650 (1999).

9. W. S. Orth and R. W. Gillham, "Dechlorination of trichloroethylene in aqueous solution using $\mathrm{Fe}^{0}$," Environ. Sci. Technol. 30, 66-71 (1996).

10. T. J. Campbell, D. R. Burris, A. L. Roberts, and J. R. Wells, "Trichloroethylene and tetrachloroethylene reduction in a metallic iron-water-vapor batch system," Environ. Toxicol. Chem. 16, 625-630 (1997).

11. B. A. Williams and T. A. Cool, "Resonance ionization spectroscopy of the chloroethylenes," J. Phys. Chem. 97, 1270-1282 (1993).

12. B. A. Williams, T. A. Cool, and C. M. Rohlfing, "Multiphoton spectroscopy of Rydberg states of tetrachloroethylene," J. Chem. Phys. 93, 1521-1532 (1990).

13. W. A. Arnold and A. L. Roberts, "Pathways of chlorinated ethylene and chlorinated acetylene reaction with Zn (0)," Environ. Sci. Technol. 32, 3017-3025 (1998). 